\title{
New Laminoplasty Technique for Preserving Paravertebral Muscles: Technical Note
}

\author{
Halil Ibrahim SECER ${ }^{1}$, Baris POLAT² \\ ${ }^{1}$ University of Kyrenia, Faculty of Medicine, Department of Neurosurgery, Kyrenia, Turkish Republic of North Cyprus \\ ${ }^{2}$ University of Kyrenia, Faculty of Medicine, Department of Orthopedics and Traumatology, Kyrenia, Turkish Republic of North Cyprus \\ Corresponding author: Halil İbrahim SECER hisecer@yahoo.com, hisecer@gmail.com, halilibrahim.secer@med.neu.edu.tr
}

\section{ABSTRACT}

AIM: To develop a new laminoplasty technique for preserving paravertebral muscles.

MATERIAL and METHODS: In this technique, semispinalis and splenius muscles are cut approximately $1 \mathrm{~cm}$ laterally to the muscle insertion point to the spinous process at the laminotomy side. Then, multifidus and rotatory muscles are dissected subperiosteally and retracted laterally. The posterior part of the spinous process is cut horizontally below the insertion point of the semispinalis and splenius muscles. At this point, all the contralateral paravertebral muscles with the cut head of the spinous process are dissected subperiosteally and retracted laterally in the same manner as the laminotomy side. After the open-door laminoplasty for reconstruction of the anatomical formation, we fixed the head of the spinous process on the opening side laminae close to the inferior part of the spinous process with a titanium wire.

RESULTS: This technique is unique in that it reconstructs the spine similar to its normal anatomical form by preserving the posterior paravertebral muscles' attachments to the bones and tension ligaments.

CONCLUSION: This technique can reduce the risk of axial pain and kyphotic deformity, and prevent the reduction of ROM.

KEYWORDS: Cervical spine, Laminoplasty, Axial neck pain, Range of motion

\section{INTRODUCTION}

L aminoplasty is an effective and safe surgical procedure for multi-segmental cervical degenerative compresJive myelopathy. The term "laminoplasty" covers many surgical procedures including the reconstruction of posterior elements of the cervical vertebrae for expanding the spinal canal. Although cervical laminoplasty is a motion preserving procedure compared to fusion surgery, several studies have reported some complications and problems such as axial neck pain, kyphotic deformity, and reduction of cervical range of motion (ROM) at long-term follow-up $(1,3,7,10,16)$. The disruption of paravertebral muscles and ligaments is the main cause of these problems, particularly neck pain. Different surgical techniques have been reported to reduce axial neck pain and restriction of cervical ROM after laminoplasty $(12,18,20)$. In this study, we report a new surgical technique for reducing these problems.

\section{MATERIAL and METHODS}

\section{Surgical Technique}

This surgical technique is useful for cervical degenerative compressive myelopathy at the level of C3-C6. If C2 and C7 decompressions are required, we make a wide hemilaminectomy at $\mathrm{C} 2$ and cranial wide hemilaminectomy at $\mathrm{C} 7$ by preserving the muscles attachments on the $\mathrm{C} 2$ and $\mathrm{C} 7$ spinous processes and limiting the damage to the attachments of the interspinous and rotator muscles with a muscle-splitting technique, as described by Shiraishi, and Shiraishi and Yato $(20,21)$.

In this technique, we decided to reconstruct the spine similar to its normal anatomical form by preserving the posterior paravertebral muscles' attachments to the bones and tension ligaments. 
After placing the patient in the prone position and then making a midline skin incision between $\mathrm{C} 2-\mathrm{C} 7$, the nuchal ligament with the trapezius muscles is divided in line with the skin incision by monopolar electrocautery (Figure 1). When we bring up the supraspinous ligaments, all the semispinalis and splenius muscles are cut approximately $1 \mathrm{~cm}$ laterally to the muscle insertion point to the spinous process at the laminotomy side. Then, the multifidus and rotatory muscles are dissected subperiosteally and retracted laterally off the inferior part of the spinous processes, laminae, and medial aspect of the facets at the laminotomy side. We stay in the natural avascular subperiosteal plane to prevent damage to the paravertebral muscles and to minimize blood loss. Also, the facet capsules must be preserved. The opening side is usually placed on the side that has worse radicular symptoms or more stenosis because it is technically easier to perform a foraminotomy to decompress the neuroforamen. The posterior part of the spinous process is cut horizontally below the insertion point of the semispinalis and splenius muscles with

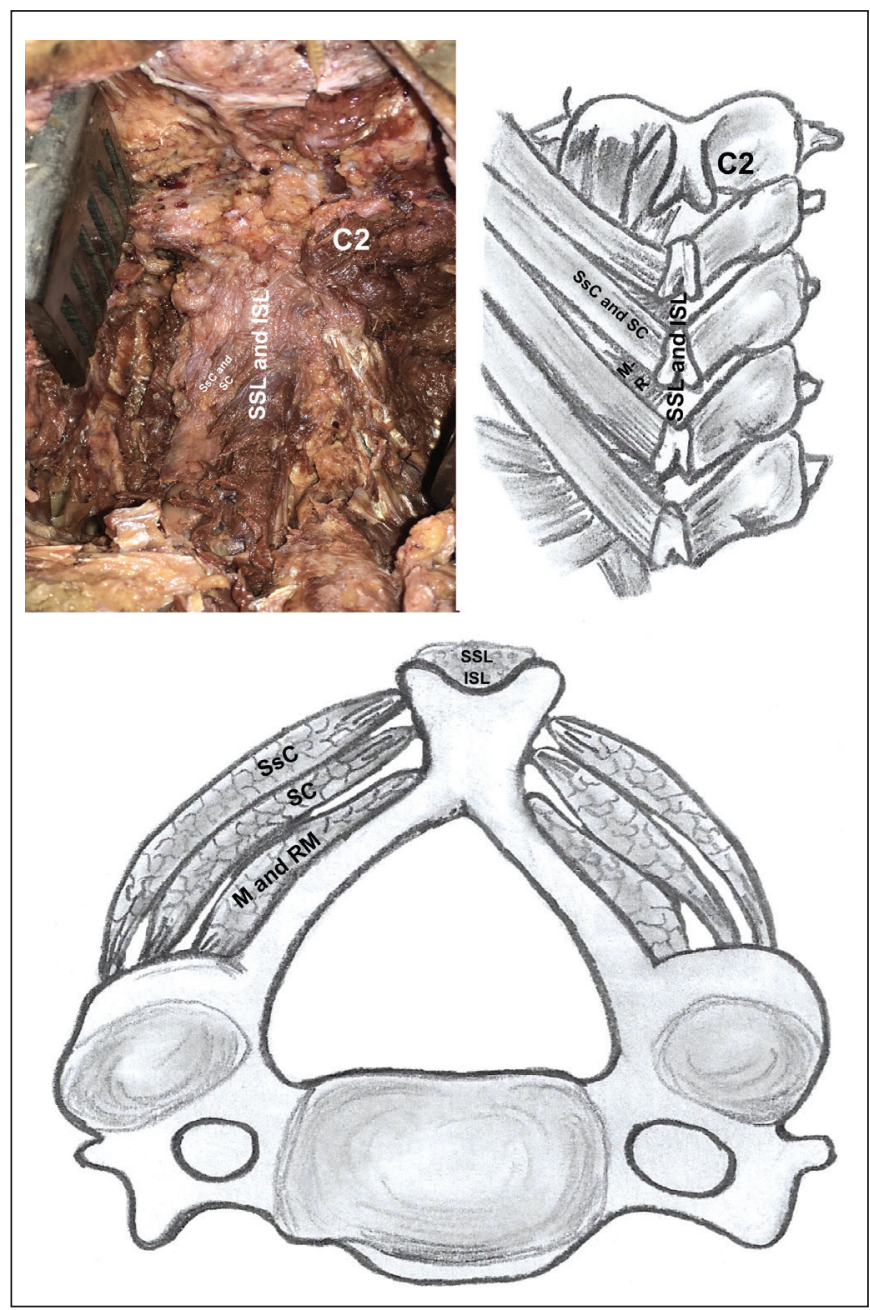

Figure 1: The paravertebral muscles at C3-C6 levels (SSL: supraspinous ligament, ISL: interspinous ligament, SsC: semispinalis capitis muscle, SC: splenius capitis muscle, M: multifidus muscle, RM: rotatory muscles). a high-speed small bone oscillating saw (Figure 2). We can keep the insertion of the semispinalis and splenius muscles at the contralateral side with this technique. We also preserve the supraspinous and interspinous ligaments at the proximal to distal extents of the exposure during this approach. At this point, all the contralateral paravertebral muscles with the cut head of the spinous process are dissected subperiosteally and retracted laterally in the same manner as the laminotomy side (Figure 2). Retractors can be placed on the sides of the wound and, if necessary, rostrally and caudally in order to facilitate visualization. The hinged side of the lamina should be cut before the laminotomy side to minimize blood loss. A4 mm diamond burr or $2 \mathrm{~mm}$ cutting burr of high speed drill is used to cut the outer cortex at the junction of lamina and lateral mass in the process of creating a green stick fracture on the hinged side, as it minimizes the risk of completely breaking through the inner cortex of the lamina. The ligamentum flavum is released with a curette at the inferior aspect of the lamina. The facets should not be violated. Subsequently, we cut the

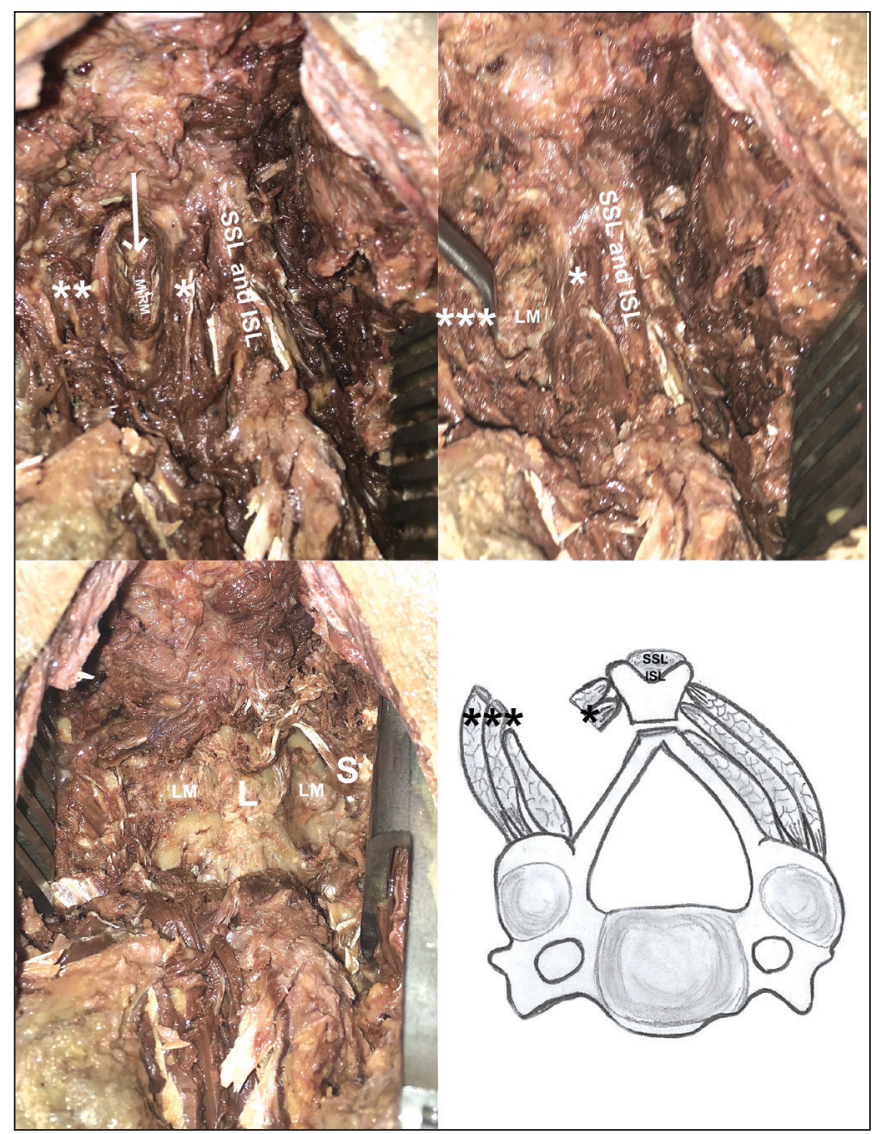

Figure 2: The arrow shows us the cutting line of semispinalis capitis and splenius capitis muscles at near the attachment of spinous process (SSL: supraspinous ligament, ISL: interspinous ligament, $\mathbf{M}$ : multifidus muscle, $\mathbf{R M}$ : rotatory muscles, $*$ : the spinous process attachment parts of the SsC and SC, **: lateral free parts of the SsC and SC, LM: medial part of the facet, L: laminae, S: the spinous process attachment parts of the SsC and SC with posterior cutting part of the spinous process and retracted of hidden side paravertebral muscles). 
lamina just medial to the pedicle with a 1 or $2 \mathrm{~mm}$ Kerrison punch and then cut the ligamentum flavum from the caudal to rostral direction at the laminotomy side. Sometimes, we use a pediatric craniotomy attachment for cutting the lamina. Epidural bleeding is controlled by bipolar electrocautery and absorbable hemostats. We place a curette under the lamina on the laminotomy side and elevate the bone. When we see the motion of the lamina, the lamina is tilted to the hinged side until the trough is closed (Figure 3). We separate the top and bottom lamina of the open side which is included in the laminoplasty from the adjacent levels with a Kerrison punch. This can be performed by gradually opening the lamina at each level and to the same degree as the other vertebrae. In this way, the gap on the laminotomy side between the lamina and facet is increased. A Woodson elevator can be used to release any adhesions between the dura and ventral lamina on the laminotomy side. Subsequently, we fix an appropriately sized allograft that is into the laminar opening with a double bent titanium miniplate of appropriate length via a center screw hole. The miniplate with allograft are placed in the laminar opening. The graft should fit securely in to the laminar gap. The miniplate is fixed with one or two $2.0 \mathrm{~mm}$ cortex screws on both the laminar and lateral mass sides (Figure 4).

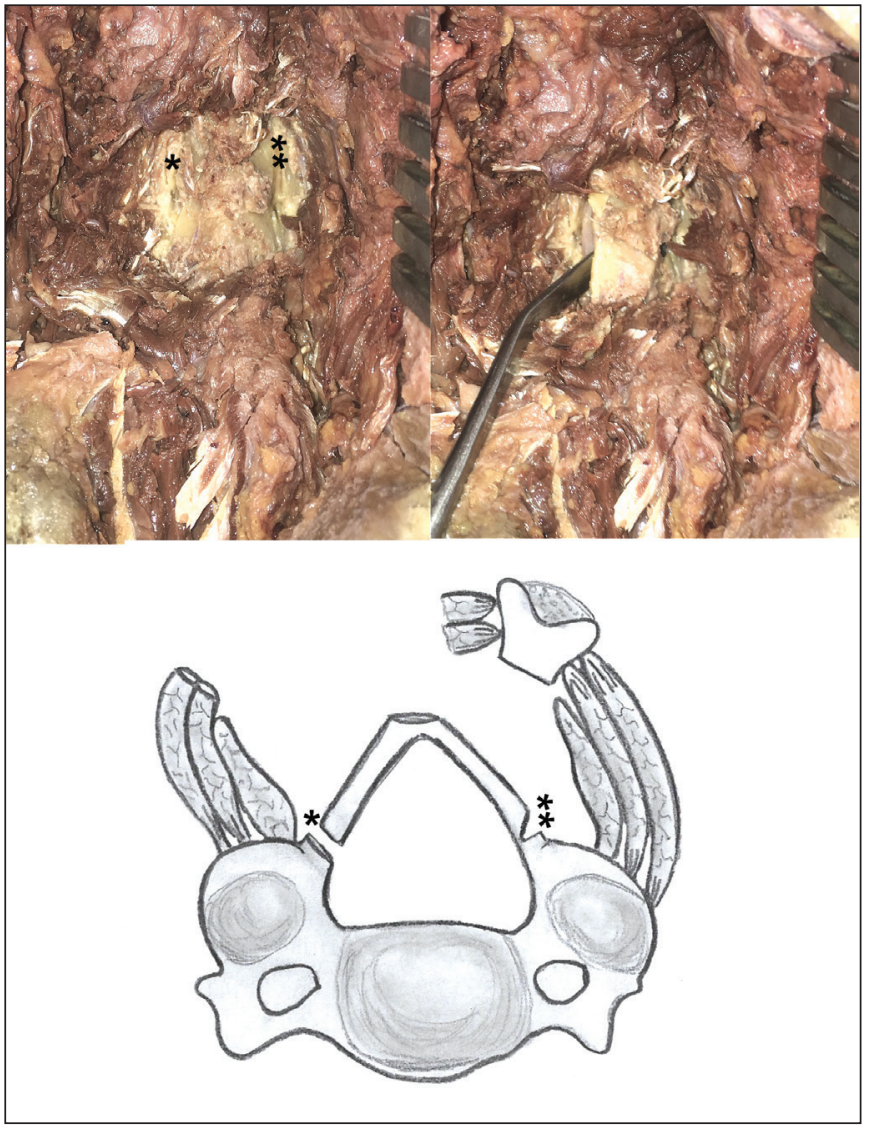

Figure 3: After cutting the laminae at the opening side and cutting the outer part of laminae at hinged side $(*$ : cutting laminae at opening side, $* *$ : thinning laminae at hinged side).
For reconstruction the anatomical formation, we make a burr at the head of the spinous process that has been previously separated and the inferior part of the spinous process at a position that is slightly lateral to the opening side. Then, we fix the head of the spinous process on the opening side decorticated laminae next to the inferior part of the spinous process with a titanium wire. We suture the semispinalis and splenius muscles with a 2/0 coated Vicryl suture at the cutting line. We close the wound by suturing layers and finish the operation (Figure 5).

\section{RESULTS}

We have performed this technique in only 2 patients in the last 3 months. The results of our cases will be published when there are sufficient number that have been completed for a sufficient follow-up period (at least 24 months), which means that we cannot share early surgical, clinical and radiological results. Although the duration of this technique is not compared with the other open-door laminectomy techniques that were previously performed, it is approximately more than 30 minutes longer for each level than the other techniques.

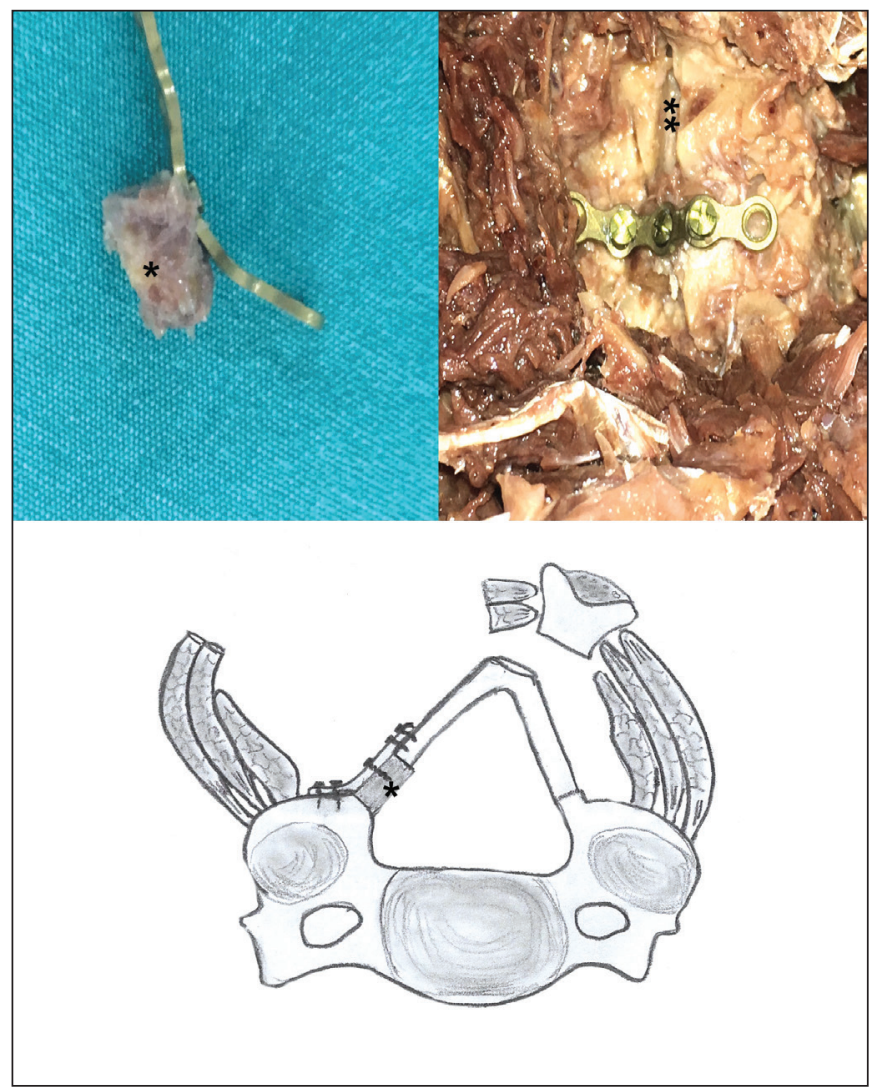

Figure 4: After the miniplate with allograft are placed in the laminar opening, the miniplate is fixed with one or two cortex screws on both the laminar and lateral mass side. (*: Autograft fixed with screw to the mini plate, $* *$ : laminar opening at the opening side). 


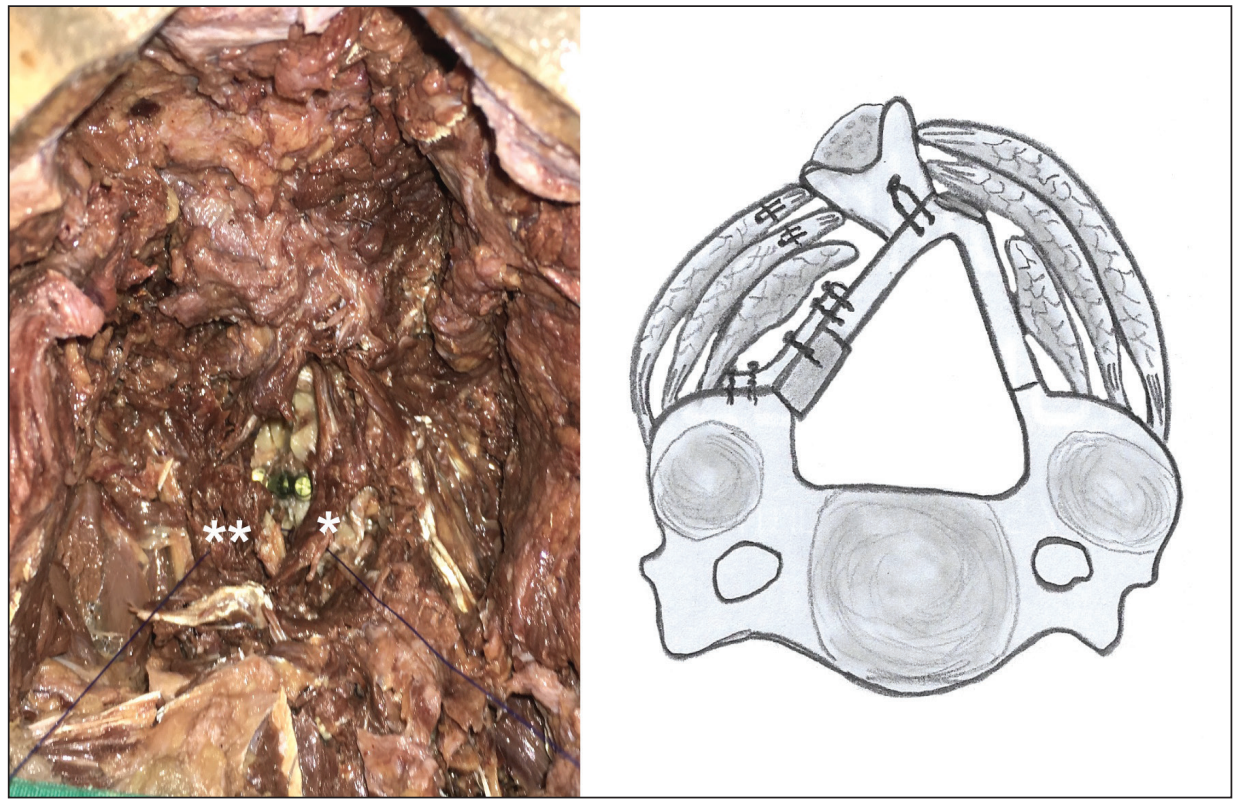

Figure 5: After fixing the head of the spinous process on top of the opening side laminae with titanium wire, suturing the semispinalis and splenius muscles at the cutting line. (*: the spinous process attachment parts of the SsC and SC, **: lateral free parts of the $\mathrm{SsC}$ and $\mathrm{SC}$ ).

\section{DISCUSSION}

The main purpose of laminoplasty techniques is to expand a narrowing spinal canal, to reconstruct the lamina and to provide stability by protecting the posterior structures as much as possible. Many techniques have been developed for this purpose that are based on open-door laminoplasty, double-door laminoplasty and muscle-sparing laminoplasty techniques $(5,13,15)$.

One of the major concerns after cervical laminoplasty is the reduction of cervical ROM. The restriction of ROM can be related to the prolonged use of a cervical collar after surgery, interlaminar bony fusion between adjacent opened lamina (19), gradual muscle and facet joint degeneration and preexisting OPLL. The axis has a key role in stabilizing and extending the head and neck. It has a large spinous process that acts as a lever arm. Five extensor muscles attached to C2 act as a dynamic stabilizer for the cervical spine. We preserved the muscle attachment at the $\mathrm{C} 2$ and $\mathrm{C} 7$ spinous process and we can reduce interlaminar bony fusion between C2-C3 and C6$\mathrm{C} 7$ with two different surgical techniques. The first technique described by Lee $\mathrm{DH}$ et al. is to perform a $\mathrm{C} 2$ laminectomy rather than a multilevel cervical open-door laminoplasty. By using this technique, interlaminar bony fusion at C2-C3 segments can be prevented and this results in better preservation of the cervical ROM while achieving similar neurological and clinical outcomes without the development of focal kyphosis or instability (14). The second surgical technique described by Suh BG et al. is to perform a wedge-shaped resection of the cranial portion of the posterior bony arch of $\mathrm{C} 7$ during opendoor laminoplasty surgery. In our technique, we perform a caudal half laminectomy to $\mathrm{C} 2$, and wedge-shaped resection to cranial portion of the $\mathrm{C} 7$ laminas in order to preserve cervical ROM (18). This preserves cervical ROM, prevents posterior bony impingement and reduces posterior neck pain (22). A randomized controlled trial demonstrated that two-weeks of neck immobilization postoperatively with a Philadelphia collar does not affect cervical pain, cervical ROM, lordotic angle and other clinical outcomes positively after cervical laminoplasty (4). A prospective study with 5 years follow up after open-door laminoplasty found that cervical ROM decreased by time until 18 months after the surgery and no further decreases occurred there after unless laminar auto-fusion was diagnosed. They found that the mean ROM of the cervical spine decreased by $38.5 \%$ at 5 years follow-up postoperatively (8).

Another major concern after cervical laminoplasty is axial neck pain. Several mechanisms are believed to be the cause of the axial pain, namely damage to the spinous process ligament-muscle complex, damage to the posterior spinal roots of C3-7, suture damage to facet joint capsules, decrease in the cervical lordotic angle and range of motion, and long-term immobilization of the neck. However, the cause of postoperative axial pain following cervical laminoplasty is still unclear and it has not been determine which posterior elements are important in reducing postoperative problems. Also, changes in the cervical alignment and muscle atrophy following operative invasion have been reported to be the main causes of axial pain $(6,9,11)$. For reducing postoperative axial pain, less invasive methods have been used, such as limited exploration or reconstruction of the posterior paraspinal muscle. Kotani et al. reported that laminoplasty with a deep extensor muscle-preserving approach appears to be effective in reducing axial pain and deep muscle atrophy as well as improving cervical spine function and the quality of life when compared to conventional open-door laminoplasty (12). Many studies have evaluated the effects of preservation of the posterior paraspinal muscles at the spinous process of $\mathrm{C} 2$ and $\mathrm{C} 7$ such as the semispinalis, which have been reported to be important as dynamic stabilizers and in their function in the posterior extensor mechanism on postoperative axial pain $(11,24)$. Our technique is a muscle-preserving selective laminoplasty technique. 
Damage to the spinous process ligament-muscle complex is another crucial factor that is thought to be related to postoperative kyphosis. Various techniques have been described to minimize disruption of the spinous process and ligament-muscle complex attachments. Preservation of the attachments of the nuchal muscles to the spinous process of the axis (the rectus major, inferior oblique, and semispinalis cervicis) and the muscle attachments to the spinous process of $\mathrm{C} 7$ (rhomboid and trapezius) protects postoperative cervical lordosis, ROM and decreases axial pain (18). The preservation of the unilateral nuchal, supraspinous and interspinous ligaments between $\mathrm{C} 2-\mathrm{C} 7$ is also important for the maintenance of cervical spinal lordosis and to lower the rate of postoperative axial neck pain (23). Reduced surgical exposure, no detachment of the muscle from the $\mathrm{C} 2$ and $\mathrm{C} 7$ spinous process and the restriction of the laminoplasty from C3 to C6 reduce postoperative kyphotic changes $(2,17)$. It has been shown in a 3D modeling study that the semispinalis muscle is significantly more stretched compared to other neck muscles for isometric neck extension. It is well recognized that the development of kyphosis and postoperative instability (25). We expose the open side lamina via cutting the splenius and semispinalis capitis muscles approximately $1 \mathrm{~cm}$ from the spinous process instead of subperiosteal dissection. In this way, we protect the attachment of the semispinalis and splenius muscles and posterior ligament-muscle complex to the spinous process, which will prevent postoperative cervical malalignment. The distance between their origins and insertions are not changed, and the muscles have almost vertical orientations. Therefore, the muscular strength necessary for extending and stabilizing the head and neck is not appreciably weakened by this operation. This technique is unique for preserving both the muscle attachments of C2, C7 and the bilateral attachments of the posterior muscle-ligament complex between C2-C7.

This surgical technique has only been performed in a small number of patients and their demographic information and clinical outcomes are not mentioned in this paper. Although the early clinical and radiological results conducted on an insufficient number of patients are satisfactory, a prospective study is required to determine the clinical and radiographic outcomes of this procedure. A prospective study will be published when this technique has been performed on a sufficient number of patients.

\section{CONCLUSION}

According to this new laminoplasty technique, we can keep the insertion of semispinalis and splenius muscles at the bilateral attachment to the spinous processes. We also preserve the supraspinous and interspinous ligaments at the proximal to distal extents of the exposure during this approach.

\section{REFERENCES}

1. Chiba K, Ogawa $\mathrm{Y}$, Ishii $\mathrm{K}$, Takaishi H, Nakamura M, Maruiwa $\mathrm{H}$, Matsumoto $\mathrm{M}$, Toyama $\mathrm{Y}$ : Long-term results of expansive open-door laminoplasty for cervical myelopathy-average 14-year follow-up study. Spine (Phila Pa 1976) 31:2998-3005, 2006
2. Duetzmann S, Cole T, Ratliff JK: Cervical laminoplasty developments and trends, 2003-2013: A systematic review. J Neurosurg Spine 23:24-34, 2015

3. Fujimori T, Le H, Ziewacz JE, Chou D, Mummaneni PV: Is there a difference in range of motion, neck pain, and outcomes in patients with ossification of posterior longitudinal ligament versus those with cervical spondylosis, treated with plated laminoplasty? Neurosurg Focus 35(1): E9, 2013

4. Hida $T$, Sakai $Y$, Ito $K$, Imagama $S$, Ishiquro N, Harada $A$ : Collar fixation is not mandatory after cervical laminoplasty: A randomized controlled trial. Spine (Phila Pa 1976) 42(5):253259, 2017

5. Hirabayashi K, Watanabe K, Wakano K, Suzuki N, Satomi K, Ishii Y: Expansive open-door laminoplasty for cervical spinal stenotic myelopathy. Spine (Phila Pa 1976) 8:693-699,1983

6. Hosono N, Yonenobu K, Ono K: Neck and shoulder pain after laminoplasty. A noticeable complication. Spine 21:19691973, 1996

7. Hosono N, Sakaura H, Mukai Y, Yoshikawa H: The source of axial pain after cervical laminoplasty-C7 is more crucial than deep extensor muscles. Spine (Phila Pa 1976) 32:2985-2988, 2007

8. Hyun SJ, Riew D, Rhim SC: Range of motion loss after cervical laminoplasty: A prospective study with minimum 5-year follow-up data. Spine J 13(4):384-390, 2013

9. Kawaguchi $Y$, Matsui $H$, Ishihara $H$, Gejo R, Yoshino O: Axial symptoms after en-bloc cervical laminoplasty. J Spinal Disord 12:392-395, 1999

10. Kawaguchi $\mathrm{Y}$, Kanamori $\mathrm{M}$, Ishihara $\mathrm{H}$, Ohmori $\mathrm{K}$, Nakamura $\mathrm{H}$, Kimura T: Minimum 10-year follow up after en bloc cervical laminoplasty. Clin Orthop Relat Res (411):129-139, 2003

11. Kato M, Nakamura H, Konishi S, Dohzono S, Toyoda H, Fukushima W, Kondo K, Matsuda H: Effect of preserving paraspinal muscles on postoperative axial pain in the selective cervical laminoplasty. Spine 33(14): E455-E459, 2008

12. Kotani $Y$, Abumi K, Ito $M$, Sudo $H$, Takahata $M$, Ohshima S, Hojo Y, Minami A: Minimum 2-year outcome of cervical laminoplasty with deep extensor muscle-preserving approach: Impact on cervical spine function and quality of life. Eur Spine J 18:663-671, 2009

13. Kurokawa T: Enlargement of the spinal canal by the sagittal splitting of spinous processes. Bessatsu Seikeigeka 2:249252, 1982

14. Lee DH, Cho JH, Hwang CJ, Lee CS, Cho SK, Ha JK: Can C3 laminectomy reduce interlaminar bony fusion and preserve the range of motion after cervical laminoplasty? Spine (Phila Pa 1976) 41(24):1884-1890, 2016

15. Mitsunaga LK, Klineberg EO, Gupta MC: Laminoplasty techniques for the treatment of multilevel cervical stenosis. Adv Orthop 2012:307916, 2012

16. Puttlitz CM, Deviren V, Smith JA, Kleinstueck FS, Tran QNH, Thurlow RW, Eisele P, Lotz JC: Biomechanics of cervical laminoplasty: kinetic studies comparing different surgical techniques, temporal effects and the degree of level involvement. Eur Spine J 13:213-221, 2004 
17. Sakaura H, Hosono N, Mukai Y, Iwasaki M, Yoshikawa H: Medium-term outcomes of C3-6 laminoplasty for cervical myelopathy: A prospective study with a minimum 5-year follow-up. Eur Spine J 20:928-933, 2011

18. Secer HI, Harman F, Aytar MH, Kahraman S: Open-door laminoplasty with preservation of muscle attachments of $\mathrm{C} 2$ and C7 for cervical spondylotic myelopathy, retrospective study. Turk Neurosurg 28(2):257-262, 2018

19. Seichi A, Takeshita K, Ohishi I, Kawaguchi H, Akune T, Anamizu Y, Kitagawa T, Nakamura K: Long-term results of double-door laminoplasty for cervical stenotic myelopathy. Spine 26:479-487, 2001

20. Shiraishi T: A new technique for exposure of the cervical spine laminae. Technical note. J Neurosurg 96 Suppl 1:122-126, 2002

21. Shiraishi T, Yato Y: New double-door laminoplasty procedure for the axis to preserve all muscular attachments to the spinous process: Technical note. Neurosurg Focus 12(1): E9, 2002
22. Suh BG, Ahn MW, Kim HJ, Yeom JS, Lee GW: Wedgeshaped resection of the posterior bony arch during open door laminoplasty to prevent postoperative motion limitation. Spine (Phila Pa 1976) 42(3):143-150, 2017

23. Sun Y, Zhang F, Wang S, Zhang L, Pan S, Yu M, Qiu S: Open door expansive laminoplasty and postoperative axial symptoms: A comparative study between two different procedures. Evid Based Spine Care J 1(3):27-33, 2010

24. Takeuchi T, Shono Y: Importance of preserving the $\mathrm{C7}$ spinous process and attached nuchal ligament in Frenchdoor laminoplasty to reduce postoperative axial symptoms. Eur Spine J 16:1417-1422, 2007

25. Vasavada AN, Li S, Delp SL: Influence of muscle morphometry and moment arms on the moment-generating capacity of human neck muscles. Spine 23:412-422, 1998 